\title{
Enteric-Coated Zoledronic Acid Tablet MER-101
}

National Cancer Institute

\section{Source}

National Cancer Institute. Enteric-Coated Zoledronic Acid Tablet MER-101. NCI

Thesaurus. Code C77855.

An oral tablet formulation containing zoledronic acid combined with a proprietary absorption enhancer for improved zoledronic acid gastrointestinal absorption with antibone-resorption activity. The third-generation bisphosphonate zoledronic acid binds to hydroxyapatite crystals in the bone matrix, slowing their dissolution and inhibiting the formation and aggregation of these crystals. This agent also inhibits farnesyl pyrophosphate synthase, an enzyme involved in terpenoid biosynthesis. Inhibition of this enzyme prevents the biosynthesis of isoprenoid lipids, donor substrates of farnesylation and geranylgeranylation during the post-translational modification of small GT Pase signalling proteins, which are important in the process of osteoclast turnover. The proprietary absorption enhancer is a GRAS (generally-recognized-as-safe) food additive. 